\title{
Variable Stars and Galactic Structure
}

\author{
Michael Feast ${ }^{1}$ and Patricia A. Whitelock ${ }^{2}$ \\ ${ }^{1}$ University of Cape Town and South African Astronomical Observatory \\ email: mwf @ast .uct.ac .za \\ ${ }^{2}$ South African Astronomical Observatory and University of Cape Town \\ email: paw@saao.ac.za
}

\begin{abstract}
Variable stars have a unique part to play in Galactic astronomy. Among the most important of these variables are the Cepheids (types I and II), the RR Lyraes and the Miras (Oand C-rich). The current status of the basic calibration of these stars in their roles as distance, structure and population indicators is outlined and some examples of recent applications of these stars to Galactic and extragalactic problems are reviewed. The expected impact of Gaia on this type of work is discussed and the need for complementary ground based observations, particularly large scale near-infrared photometry, is stressed.
\end{abstract}

Keywords. Distance Scale, Variable Stars, Galactic Structure

\section{Introduction}

Gaia will obtain parallaxes, proper motions, radial velocities and metallicities $([\mathrm{Fe} / \mathrm{H}])$ for a vast number of stars and other objects of all types, and these data will tell us much about our own Galaxy. One might ask why we should be particularly interested in the Gaia data for pulsating variable stars so far as Galactic composition, structure and dynamics are concerned. In the present review we discuss the importance of some major classes of pulsating variables, classical Cepheids, RR Lyrae variables, type II Cepheids and Miras. These variables, once their luminosities are calibrated by Gaia, will provide accurate distances both within our Galaxy and beyond, reaching beyond the distances achieved by Gaia itself. We will also point out some of the special problems that these stars pose for Gaia.

\section{Gaia parallaxes and their use}

Table 1 gives the expected accuracy $(\sigma)$ of the absolute magnitudes determined for single relatively bright stars $(6<V<12 \mathrm{mag})$ and assuming that for such stars $\sigma_{\pi}=$ $10 \mu a s$ (see ESA Gaia web page). The table also gives an estimate of the statistical bias expected in the absolute magnitude calibration (Lutz-Kelker type bias). These values of the bias are small. However, they are significant if one is aiming to obtain distance scales with an accuracy of 2 percent or better $(0.04 \mathrm{mag})$. Such accuracies are already being claimed for the distance modulus of the LMC (0.04 mag from eclipsing binaries (Pietrzyński et al. 2013) and as small as 0.03 mag for classical Cepheids, if metallicity effects are ignored). Furthermore, these bias corrections are asymmetrical and have large uncertainties (Koen 1992) due to their statistical nature and to the assumptions made in estimating them. Thus it is unlikely that stars in a given sample are always selected on the basis of parallax alone or that they are drawn from a population distributed uniformly in space. Note also that the bias correction required depends on the use to which the parallaxes are to be put (e.g., estimating distance moduli or estimating distance (see, e.g., Feast 2013). It is interesting to note that some VLBI parallaxes of star forming regions 
Table 1. Accuracy of Gaia parallaxes.

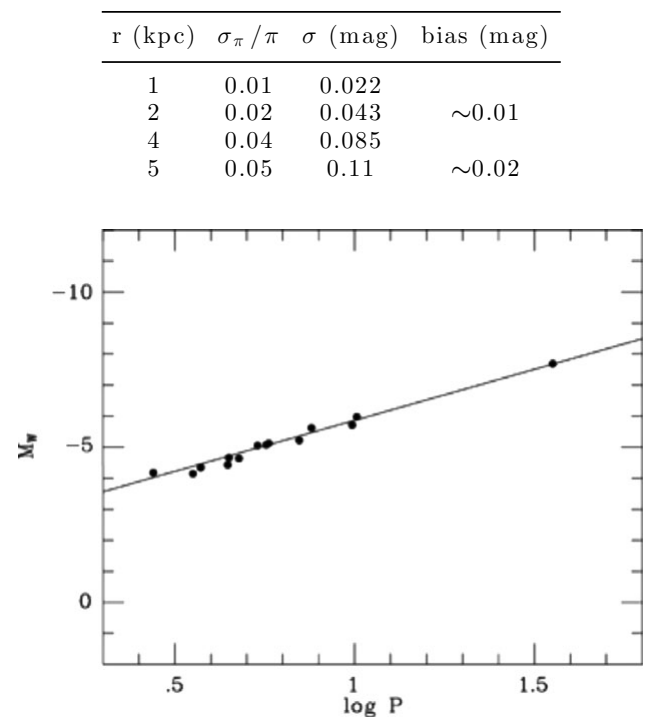

Figure 1. Reddening-free PLC relation for Cepheids with good HST and Hipparcos Parallaxes (Van Leeuwen et al. 2007).

(e.g., Reid et al. 2009) have percentage accuracies similar to those expected from Gaia and are presumably subject to bias (Stepanishchev \& Bobylev 2013) though the effect of this on derived Galactic constants is probably complex (see Bovy, these proceedings). These considerations are important for the use of Gaia parallaxes in calibrating luminosities. In the next few sections we summarize the present status of the luminosity calibration of some well known variable stars and indicate what advances we can expect from Gaia.

\section{Classical Cepheids}

Fig. 1 shows a reddening-free period-luminosity-colour (PLC) relation in $V, I\left(P W_{V I}\right)$ for classical Cepheids with the best HST and Hipparcos parallaxes (Benedict et al. 2007, van Leeuwen et al. 2007). The slope of this relation is close to that found for LMC Cepheids, which are metal-deficient with respect to those in the solar neighbourhood. However, the slope evidently depends rather critically on one point. Also, both in the Galaxy and beyond, the long-period Cepheids, being bright, are important. If it is assumed that the Galactic relation has the same slope as that found in the LMC, then a Galactic zero-point can be found from HST and Hipparcos parallaxes using the method of reduced parallaxes. This avoids some of the bias problems just discussed (see e.g., Feast 2002, 2013). In this way a zero point with a standard error of $0.03 \mathrm{mag}$ is obtained. This assumes the LMC slope. The effects of metallicity on this slope and the zero point are at present poorly understood though they are probably small.

How will Gaia help clarify this situation?

For a sample of $32 \mathrm{LMC}$ Cepheids with $1.0<\log P<1.7$, reddening-free $W_{V I}-\log P$ and $W_{J K_{S}}-\log P$ relations show a dispersion (per star) of $\sigma=0.175$ mag and $0.085 \mathrm{mag}$ respectively (Feast et al. 2012). The scatter may have a component due to the thickness of the LMC, but is probably mainly observational: that in $V I$ being greater due to the coefficient of the colour term in $V I$ being numerically larger. In using Gaia data on Cepheids to calibrate the $P W$ relations it is obvious that one must aim to keep bias 


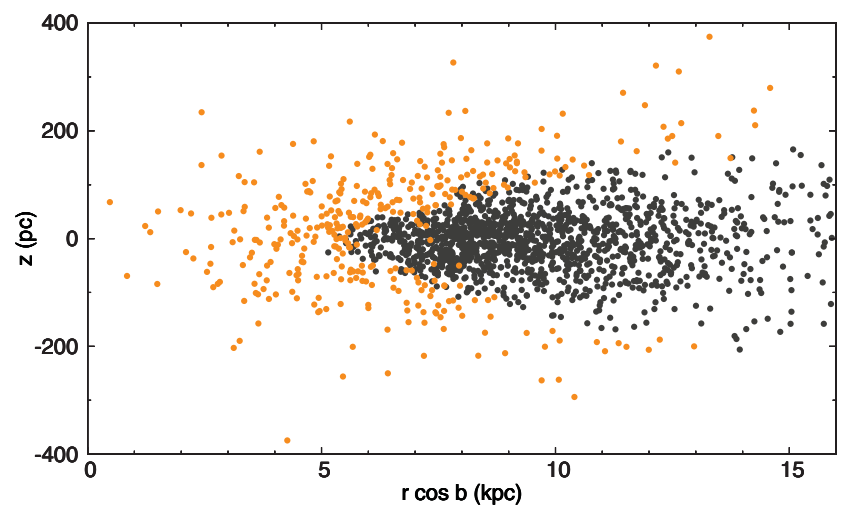

Figure 2. The simulated inner Galaxy $\left(|l|<5^{\circ}\right)$, with projected distance to the Sun plotted versus height above the Galactic plane. The light dots correspond to Cepheids that will be observable by Gaia $(G<20)$ and the dark dots correspond to Cepheids that are too faint to be observed by Gaia $(G>20)$ (Windmark et al. 2011).

effects very small and it is also desirable that the individual uncertainties are if possible considerably smaller than the LMC scatter just quoted. As a rough guide Table 1 suggests that one should be limiting oneself to Cepheids within about $2 \mathrm{kpc}$. With this restriction we can expect many Cepheids with periods less than 10 days with $\sigma_{\pi} / \pi \lesssim 0.02$, perhaps about 20 with the same uncertainty and periods over 10 days, and rather few in this category with periods over 20 days. Of course, all this may change when the actual performance of Gaia is known, but it suggests that at the shorter periods there will be sufficient data to study the various Cepheid relations in some detail, including estimates of their intrinsic scatter. At the longest periods we may be forced to employ the method of reduced parallaxes on more distance samples, possibly grouping the stars according to period.

The scatter in the $\mathrm{LMC} W_{J K_{S}}-\log P$ relation given above is obviously a maximum value for the intrinsic scatter. This being the case Table 1 suggests that beyond about $4 \mathrm{kpc}$ this relation (calibrated by Gaia) will provide better distances for individual Cepheids than the Gaia parallaxes themselves, again provided the metallicity effects are negligible or can be quantified. This is important since near-infrared $\left(J H K_{S}\right)$ observations of Cepheids have, potentially, a much wider reach over our Galaxy than Gaia itself. Fig. 2 illustrates this point. It shows a likely distribution of Cepheids in a direction toward the Galactic centre. The dark points are Cepheids which will not be observable by Gaia, primarily due to interstellar extinction. However, they will be well within the range of current near-infrared facilities. Note that the 20 day Cepheids found by Matsunaga et al. (2011) in the Galactic centre have $A_{V} \sim 25$ mag but are still quite bright in the infrared $\left(K_{S} \sim 10 \mathrm{mag}\right)$. This highlights the importance of ground-based near-infrared photometry of Cepheids. This together with Gaia results on the $W_{J K_{S}}-\log P$ zero-point, intrinsic scatter and (hopefully) metallicity effects, will allow the distribution (and with (infrared) velocities) the kinematics of Cepheids over much of the Galactic disc to be determined. Such studies can be made as a function of age, since Cepheid age is related to period: $\sim 25 \mathrm{Myr}$ at 20 days, $\sim 65 \mathrm{Myr}$ at 6 days (Bono et al. 2005 ).

\section{RR Lyrae Variables}

The RR Lyrae variables are indispensable tracers of, and distance indicators for, old stellar populations in our own and nearby galaxies. They are of current importance in two 


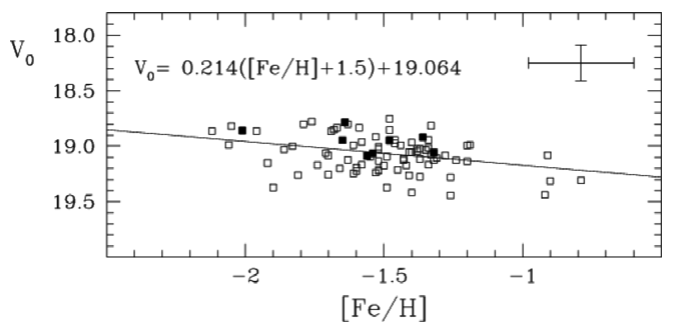

Figure 3. Reddening corrected mean magnitude of the RR Lyrae variables in two LMC fields as a function of the metallicity $[\mathrm{Fe} / \mathrm{H}]$. Filled symbols are double-mode pulsators; open symbols ab- and c-type RR Lyrae stars (Gratton et al. 2004).

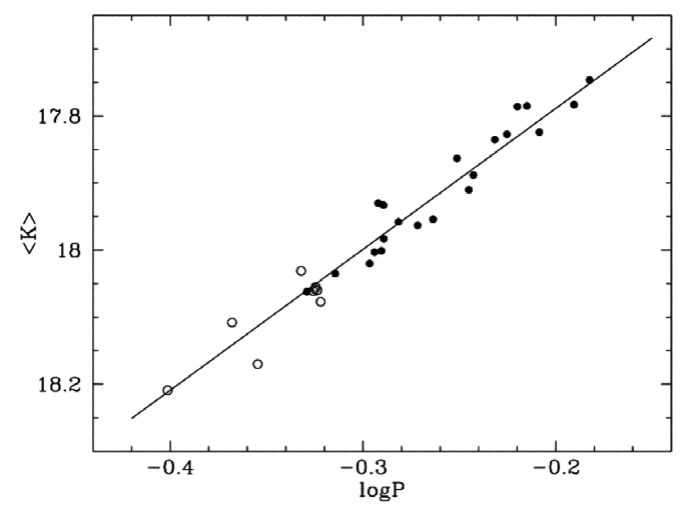

Figure 4. The PL $(K)$ relation for the Reticulum RR Lyrae stars (Dall'Ora et al. 2004).

major areas in our own Galaxy: the distances and hence the ages of globular clusters, and the structure and kinematics of the Galactic halo and its implication for galaxy formation. Two areas of research on the Galactic halo are, first, the nature, extent and significance of infalling streams, and second, the general overall structure of the halo. As regards the latter point, there is a current debate as to whether or not the halo consists of two major components: an inner, prograde halo and an outer, retrograde halo. The evidence for a two component model has been summarized by Carollo et al. $(2007,2010)$ and by Beers et al. (2012), but it has been criticized by Schönrich et al. (2011) primarily on the basis of the distances adopted by Carollo/Beers.

There are two favoured ways of using RR Lyraes for distance determination. The first is a $V-[\mathrm{Fe} / \mathrm{H}]$ relation. Perhaps the best example of this is from the LMC (Fig. 3). As can be seen the scatter is quite large. Much of this is probably due to the depth of the LMC halo though uncertainties in reddening corrections and in $[\mathrm{Fe} / \mathrm{H}]$ values may contribute. At present the intrinsic width of the relation is not well determined. The second is an $M_{K_{S}}-\log P$ relation. This is very promising as can be seen from Fig. 4 , which shows the relation for the LMC globular cluster Reticulum (Dall'Ora et al. 2004). The scatter per star is very low $(0.03 \mathrm{mag})$. The dependence of the zero-point of this relation on $[\mathrm{Fe} / \mathrm{H}]$ is not well determined, but may well be quite small (see Feast 2013 for a discussion).

The current calibration of the two relations from the HST parallaxes of five nearby RR Lyraes (Benedict et al. 2011) gives the following zero-points:

$M_{V}=0.46 \pm 0.03 \mathrm{mag}$ at $[\mathrm{Fe} / \mathrm{H}]=-1.5$, $M_{K_{S}}=-0.54 \pm 0.03 \mathrm{mag}$ at $\log P=-0.28$. 

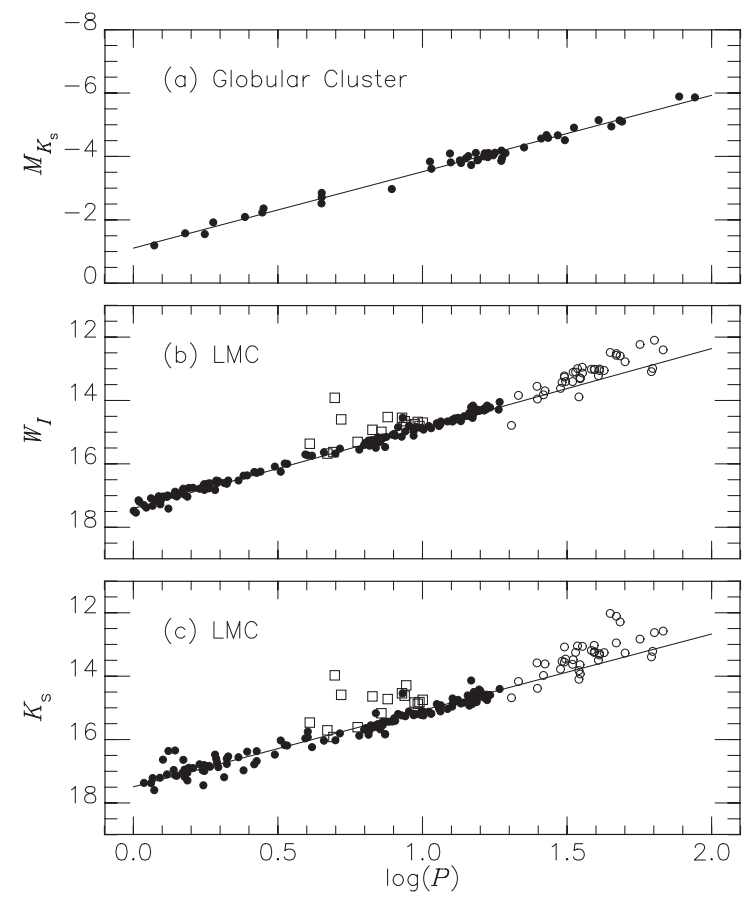

Figure 5. PL relations for type II Cepheids (see text for details) from Feast (2013).

Whilst this calibration seems rather secure and, for instance, leads to a distance modulus near $18.5 \mathrm{mag}$ for the LMC in agreement with several other indicators, statistical parallax work gives absolute magnitudes which are about a third of a magnitude fainter. The differences $(\Delta)$ are:

$\Delta M_{V}=0.33 \pm 0.13 \mathrm{mag}$ (Gould \& Popowski (1998) and earlier papers),

$\Delta M_{K}=0.37 \pm 0.08 \mathrm{mag}$ (Dambis 2009).

This is an 18 percent difference in the distance scale. If the statistical parallax result were used it would imply an LMC modulus near $18.2 \mathrm{mag}$, and a distance to the Galactic centre near $7 \mathrm{kpc}$, in contrast to most other estimates which are near $8 \mathrm{kpc}$ (see, e.g., Feast (2013) for a discussion of LMC and Galactic centre distances). This difference is not understood at present. There seems a possibility that the statistical parallax result might be affected by the relatively simple model of the halo assumed.

Clearly Gaia parallaxes will place the calibration on a much improved footing. There are many RR Lyraes with $6<V<12$ mag and distances less than about $2 \mathrm{kpc}$. Thus Gaia parallaxes should provide excellent calibrations for both $V$ and $K_{S}$ relations including estimates of their intrinsic scatter and any metallicity effect on the $K_{S}$ relation. This will, however, require good infrared light-curves for the calibrators. In addition good values of $[\mathrm{Fe} / \mathrm{H}]$ will be required, either from Gaia itself or from the ground.

Provided any metallicity effect on the $K_{S}-\log P$ relation is determined (using Gaia data) and the low scatter in this relation, just mentioned, is confirmed, this relation should give better distances than the Gaia parallaxes themselves for RR Lyraes further away than about $2 \mathrm{kpc}$. It will then be possible to carry out extensive studies of the distribution (and kinematics) of RR Lyraes over a large volume (note that an RR Lyrae with a period of 0.5 days has $K_{0} \sim 14.5 \mathrm{mag}$ at $10 \mathrm{kpc}$.) This will require extensive $J(H) K_{S}$ work over the whole sky, but this seems entirely feasible. 


\section{Type-II Cepheids}

A brief mention should be made of the type II Cepheids. These lie in the same period range as classical Cepheids, but are low-mass objects belonging to the halo, including globular clusters, as well as to the old and/or thick disc populations. They are often divided into groups according to period: BL Her stars $(P<4$ days $)$, W Vir stars $(4<$ $P<20$ days $)$ and RV Tau stars $(P>20$ days $)$. Their potential importance as distance indicators has only recently been fully recognized. This is due primarily to the work of Matsunaga et al. (2006) on type II Cepheids in globular clusters and the LMC work of the OGLE group (e.g., Soszyński et al. 2008). In globular clusters there is a good $K_{S}-\log P$ relation (see Fig. 5a), which may extend to the RR Lyrae stars. (e.g., Matsunaga et al. 2006; Benedict et al. 2011). The LMC results (in $K_{S}$ and $W_{V I}$, Fig. 5 b and c) are broadly in agreement with the globular clusters and the low scatter suggests that any metallicity effect is small. However, the LMC work reveals a number of complications. In the mean the longer period LMC stars lie above a linear PL relation and there are a significant number of stars above the PL relations at intermediate periods. These "peculiar W Vir" stars are binaries and can be recognized by their light curves. It is of some interest that, whilst binaries among Classical Cepheids are known in both our Galaxy and the LMC, there are only a few examples of LMC classical Cepheids deviating significantly from PL relations (e.g., Soszyński et al. 2008).

At present the zero-points of Type II Cepheid relations have to be estimated assuming the distances to globular clusters or to the LMC, or using pulsation parallaxes. Gaia should allow direct calibration from parallaxes.

\section{Mira Variables}

This and the following sections discuss Mira variables, which are large amplitude pulsators at the tip of the AGB. The class contains both O-rich and C-rich stars, the latter due to stellar dredge-up processes. There is currently a great deal of interest in these stars and Gaia should enable major advances in understanding them and in using them to study Galactic structure.

Much of the current interest is related to new mid- and far-infrared observations (e.g., using the Herschel and Spitzer spacecraft) and to the study of mass loss and dust formation which are not quantitatively understood. This is crucial for our understanding of stellar evolution and the composition of the interstellar medium, especially at early times. At the same time, interferometry is providing new insights on the diameters and overall shapes of these stars as well as on their surface non-uniformities.

It is known from the LMC that both O- and C-rich Miras follow similar infrared PL relations (Fig. 6). These relations show a scatter (per star) of 0.14 mag which is clearly an upper limit to the intrinsic scatter. Using Hipparcos parallaxes alone the zero-point for the O-rich stars is obtained with an uncertainty of $\pm 0.10 \mathrm{mag}$, while including other methods (Whitelock et al. 2008) brings this down to \pm 0.07 mag. Since there are large numbers of Galactic Miras (the GCVS gives a very incomplete list of about 8000) Gaia should in principle make a significant improvement on this calibration. However, these stars present some serious, and unique, challenges to Gaia, as outlined below.

\subsection{The problem of Mira amplitudes and periods}

All Miras have large ranges in visual magnitude; they are defined as having photographic or $V$ changes in excess of 2.5 mag (though some stars with smaller amplitudes are similar to Miras) and the range can exceed $10 \mathrm{mag}$ for very red stars. Mira itself, o Ceti, has a range of over 6 mag (e.g., Barthès \& Mattei 1997). This will mean that some of the 


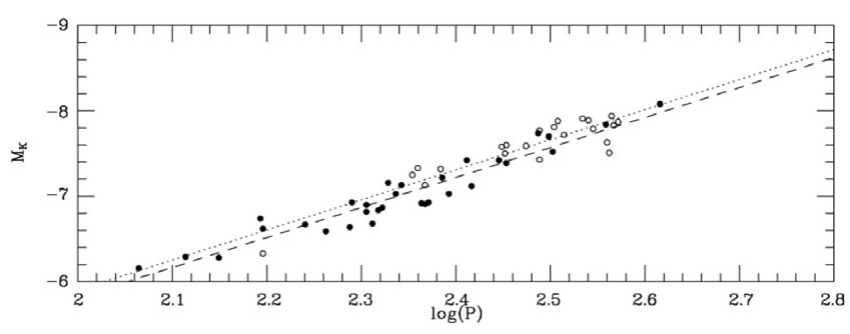

Figure 6. PL $(K)$ relation for LMC Miras from Whitelock et al. (2008). Open and closed symbols represent $\mathrm{O}$ - and C-rich Miras, respectively.

variables will be either too bright or too faint to be accurately observed by Gaia at some phases. For instance, whilst the GCVS lists 1883 Miras which are brighter than visual or photographic magnitude 12 at maximum, only 18 lie in the range 6 to 12 mag at all phases, the range for the most accurate parallaxes (see Table 1). Of these, eleven are Orich and seven C-rich and have periods in the range 200 to 450 days. Additionally, whilst the periods of Miras are typically in the range 100 to 1000 days, the period distribution in the solar neighbourhood peaks between 200 and 400 days and there are many Miras with periods near one year which will also present problems given the baseline for parallax measurement. Hopefully, observations over the life of the Gaia mission will overcome or at least ameliorate these problems.

\subsection{The problem of Mira sizes}

It has long been known that the angular sizes of Miras are large and depend on the wavelength at which they are measured. This is due to the great depth of the atmosphere and the variation of opacity with wavelength. For instance speckle interferometry showed that for O Miras, the diameter in the TiO bands was double that in the nearby continuum (Labeyrie et al. 1977). The size is a minimum in the near infrared, but even there it will be greater than the parallax, e.g., for $o$ Ceti, the Hipparcos parallax is $9.3 \pm 1$ mas and the angular diameter at $2.2 \mu \mathrm{m}(K)$ is 24 mas (Mennesson et al. 2002). Early interferometric work (e.g., Lattanzi et al. 1997) showed that these stars are not well represented by uniform circular discs. In the case of C Miras, near-infrared photometry (Whitelock et al. 1997, 2006, Feast et al. 2003) provided evidence that some of these stars produce dust erratically as puffs in random directions, after the manner of $\mathrm{R} \mathrm{CrB}$ stars and possibly connected with large convection cells. Advances in interferometric techniques and high resolution imaging (e.g., Fig. 7) have confirmed this. It seems that, at least for dusty Miras, there may be no clear centre of light and that bright (low circumstellar absorption) patches may move around in ways not simply related to the pulsation.

It is clear that these effects as well as those mentioned in the last section will complicate the analysis of the astrometry. However, given the wealth of the expected Gaia data, we may hope that it will offer new insights into mass loss and convection in these stars, which are at present very poorly understood, as well as giving us valuable parallaxes, proper motions and radial velocities.

\section{Miras as Age Indicators}

It has been known for some while now that the period of a Mira is a measure of its age, the relation being similar for O- and C-rich Miras. The main evidence for this is the relation between kinematics and period, as well as the Miras in Galactic globular clusters and in intermediate age Magellanic Cloud clusters (see Feast (2009) for a summary). We 


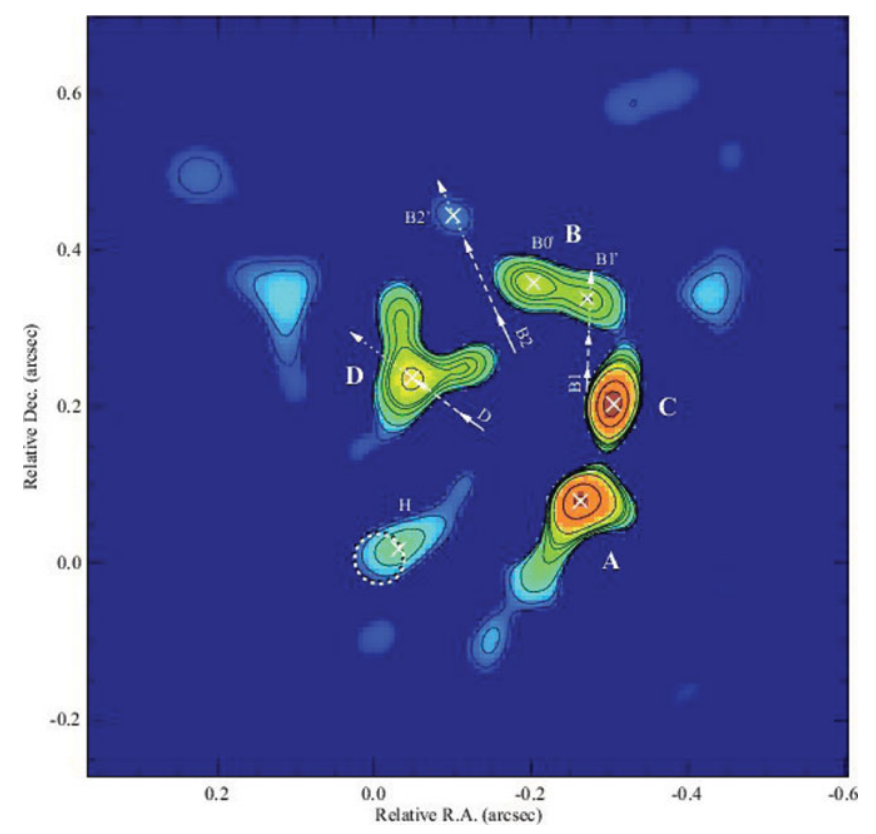

Figure 7. $H$-band image of the C-rich Mira CW Leo (IRC+10216) from Leão et al. (2006), showing only the highest spatial frequencies of the brightness.

can estimate rough ages of $\sim 12$ Gyr for 200 day Miras and $\sim 2$ Gyr at 500 days. The very long period $\mathrm{OH} / \mathrm{IR}$ Miras must be even younger and more massive.

An interesting case in which this age/mass and period relation appears to be violated is that of the 551-day Mira in the centre of the globular cluster Lyngå 7. This object which is known to be C-rich (in contrast to Miras in other Galactic globular clusters) was investigated by Feast et al. (2013) who established that it was a radial velocity member. Its initial mass is expected to have been $\sim 1.5 M_{\odot}$, i.e, about twice the turn-off mass in the cluster. We suggested that it is the evolutionary product of a stellar merger. At the cluster distance we find that it has $M_{b o l}=-5.0 \mathrm{mag}$, in excellent agreement with the predictions from the PL relation of $M_{b o l} \sim-5.2 \mathrm{mag}$. This is particularly interesting as the first example of the PL relation applying to star formed by merging two others. Such mergers are likely to be important in dense environments, such as the bulge (see below).

\section{Miras and Galactic Structure}

Miras have an important role to play in understanding the Galactic bulge and other Galactic structures. Early evidence for a bar-like structure in the bulge, with hints of complex underlying structure, was provided by the distribution of Miras on either side of the Galactic Centre (Whitelock \& Catchpole 1992). Large scale, near-infrared studies of the bulge region should enable this work to be carried much further.

As regards the ages of stars in the bulge the Miras have long presented a puzzle. On the basis of colour-magnitude diagrams it has been claimed that the bulge population, excluding the central regions, is very old (see e.g., Rich 2013 and references therein). However, the bulge contains $\mathrm{O}$ Miras with a large range of periods, up to more than 700 days (Whitelock et al. 1991), suggesting a significant intermediate age population. It was suggested long ago (Greggio \& Renzini 1990) that these long period Miras might 


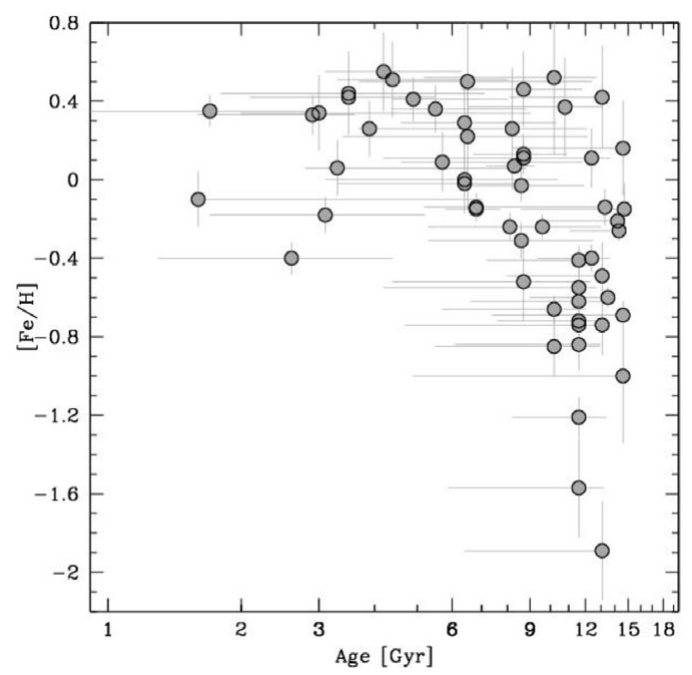

Figure 8. $[\mathrm{Fe} / \mathrm{H}]$ as a function of age for dwarfs in the Galactic bulge (Bensby et al. 2013) illustrating the presence of a young population.

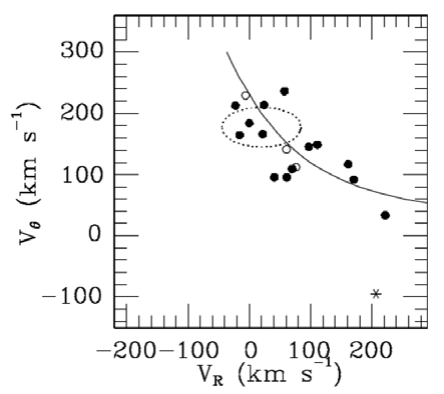

Figure 9. Space motions of Miras in the Solar neighbourhood with $145<P<200$, illustrating the possible influence of the bar (Feast 2003).

be formed by mergers. The discovery of blue stragglers in the bulge and the suggestion that they represent an important population there (e.g., Clarkson et al. 2011) makes this suggestion more plausible. However, it has also been recently suggested that the bulge does contain an intermediate age and relatively metal-rich population (Bensby et al. 2013, see Fig. 8). Evidently the question of the origin of long period Miras in the bulge needs a quantitative discussion based on much more data. Note that the results discussed in section 7 suggest that Miras may remain good distance indicators even if they result from mergers.

Analysis of the space motions of the short period (145-200 day) Miras (i.e. a globular cluster-age population) in the general Solar neighbourhood (Feast \& Whitelock 2000) gave the result shown in Fig. 9. This shows that all these Miras with velocities in the direction of Galactic rotation $V_{\theta} \lesssim 160 \mathrm{~km} \mathrm{~s}^{-1}$ have positive (outward) velocities $\left(V_{R}\right)$ in the Galaxy. It was suggested at the time that this was related to a bar-like structure. Gaia together with extensive near-infrared photometry will allow the Galactic kinematics of Miras of all periods to be studied in detail out to large distances. It will then be interesting to see whether the hint of structure given by Fig. 9 is confirmed and whether it can be fitted with the more complex structure of the bulge, perhaps an X-shape, which is now 

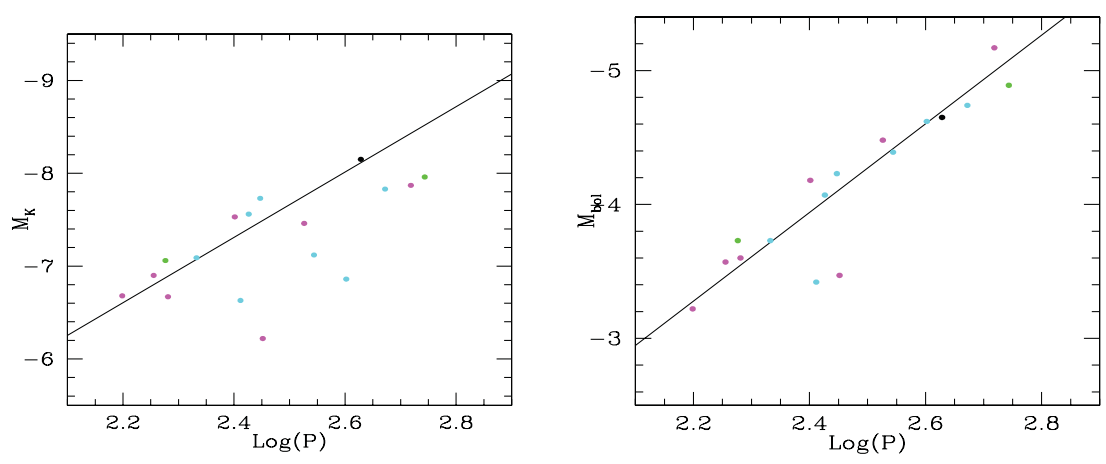

Figure 10. The PL relations for Local Group dwarf spheroidal galaxies, symbols as follows: Fornax-cyan; Leo I-magenta; Sculptor-green; Phoenix-black.

being proposed (Vásquez et al. 2013) and which the Mira data also hinted at (Whitelock \& Catchpole 1992).

\section{Miras and the Extragalactic Distance Scale}

The importance of classical Cepheids for the distance scale is well known and in recent times the value of these distance indicators in the near- and the mid-infrared has been stressed in view of the expected capabilities of JWST. The usefulness of Miras at infrared wavelengths is less well known. However, these stars are bright at infrared wavelengths: At $K(2.2 \mu m)$ :

A 50 day Cepheid has $M_{K} \sim-7.9 \mathrm{mag}$

A 380 day Mira has $M_{K} \sim-7.9 \mathrm{mag}$

At $8 \mu m$ :

A 50 day Cepheid has $M_{8} \sim-8.3 \mathrm{mag}$

A 230 day Mira has $M_{8} \sim-8.3 \mathrm{mag}$

A 380 day Mira has $M_{8} \sim-9.2 \mathrm{mag}$

Miras have the additional importance of having a range of ages. They are thus observable in populations devoid of young stars, e.g., elliptical galaxies, and since they will be less concentrated to the planes of galaxies they will be easier to resolve at large distances.

Figs. 10 and 11 illustrate the use of Miras as distance indicators in the Local Group. Fig 10(a) shows a plot of $M_{K}$ for Miras in a number of Local Group dwarf spheroids whose distances were derived by other methods (see Menzies et al. $(2008,2010,2011$ ) and Whitelock et al. (2009)). These Miras are all, to the best of our knowledge, carbon-rich. As will be seen there is considerable scatter, many stars falling below the line derived from LMC C Miras as a consequence of circumstellar extinction. However, as Fig. 10(b) shows, a much clearer relation is obtained if one uses bolometric magnitudes. The bolometric corrections (BC) were derived from a colour-BC relation. As will be seen, two stars still fall below the LMC relation. This is due either to the stars undergoing an RCB-like obscuration event (see above) or to the derived bolometric corrections being incorrectly estimated for these highly reddened short-period $\mathrm{C}$ Miras which appear to be unique to the dwarf spheriodals. The latter idea is supported by the much brighter bolometric magnitude derived for the variable in Fornax by Sloan et al. (2012) using Spitzer data.

Fig. 11 shows the Mira PL relations in the the Local Group dwarf irregular NGC6822 (Whitelock et al. 2013). Most of the Miras are carbon-rich and it can be seen that the derived bolometric magnitudes fit a PL relation much better than do the $K$ magnitudes. A few O-rich Miras are also plotted. At the long periods these tend to lie above the PL 

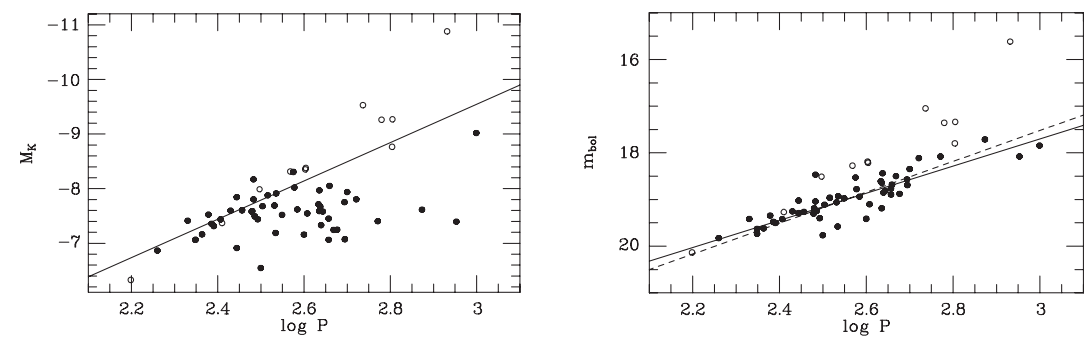

Figure 11. The PL relations for NGC6822 from Whitelock et al. (2013). Open and closed circles are O-rich and C-rich Miras, respectively. In the right hand figure the dashed line has the slope of the LMC PL relation, while the solid line was derived from the C stars in NGC 6822.

relation both at $K$ and $m_{\text {bol }}$. These may be hot bottom burning stars and/or overtone pulsators.

It will be clear from the above that in using Miras as distance indicators there are a number of possible problems to be kept in mind. As for other distance indicators, including Cepheids, single objects may give deviant results.

\section{Summary}

The discussion of sections 1 to 5 suggests that once firmly calibrated by Gaia, nearinfrared period-luminosity relations of Classical and Type II Cepheids and RR Lyrae variables can be used to extend understanding of our own and nearby galaxies, far beyond the reach of Gaia itself.

There can be no doubt that Gaia observations of Mira variables will provide tremendous new insights into the kinematics of different Galactic populations, particularly if we can separate these stars according to period. The calibration of Mira PL relations will be important for both Galactic and extragalactic work. It also seems likely that detailed Gaia observations of a few nearby Miras together with ground based observations, including interferometry, will revolutionize our understanding of Mira surface structure, of what is actually happening during pulsation cycles and of how mass loss is driven.

However, to fully exploit the Gaia results for all the classes of variables discussed in this review, extensive near-infrared photometry (e.g., $J H K_{S}$ or similar) will be required.

\section{Acknowledgements}

The authors gratefully acknowledge the receipt of research grants from the National Research Foundation (NRF) of South Africa and thank John Menzies for his corrections.

\section{References}

Barthès, D., \& Mattei, J. A. 1997, AJ, 113, 373

Beers, T. C., et al. 2012, ApJ, 746, 34

Bensby, T., et al. 2013, A\&A, 549, 147

Benedict, G. F., et al. 2007, AJ, 133, 1810

Benedict, G. F., et al. 2011, AJ, 142, 187

Bono, G., et al. 2005, ApJ, 621, 966

Carollo, D., et al. 2007, Nature, 450, 1020

Carollo, D., et al. 2010, ApJ, 712, 692

Clarkson, W. I. et al. 2011, ApJ, 735, 37

Dambis, A. K. 2009, MNRAS, 396, 553

Dall'Ora, M., et al. 2004, ApJ, 610, 269 
Feast, M. W., \& Whitelock, P. A. 2000, MNRAS, 317, 460

Feast, M. W. 2002, MNRAS, 337, 1035

Feast, M. W., et al. 2003, MNRAS, 346, 878

Feast, M. 2003, Mass-losing pulsating stars $\&$ their cs matter, Dordrecht: Kluwer, p. 83

Feast, M. W. 2009, AGB Stars and Related Phenomena, p. 48

Feast, M. W. et al. 2012, MNRAS, 421, 2998

Feast, M. W. et al. 2013, MNRAS, 428, 36

Feast, M. W. 2013, Planets, Stars and Stellar Systems Vol. 5, Dordrecht: Springer, p. 829

Gould, A., \& Popowski, P. 1998, ApJ, 508, 844

Gratton, R. G., et al. 2004, A.\&A., 421, 937

Greggio, L., \& Renzini A. 1990, ApJ, 364, 35

Koen, C. 1992, MNRAS, 256, 65

Labeyrie, A., et al. 1977, ApJ, 218, 75L

Lattanzi., M. G., et al. 1997, ApJ, 485, 328

Leão, I. C., et al. 2006, A.\&A., 455, 187L

Matsunaga, N., et al. 2006, MNRAS, 370, 1979

Matsunaga, N., et al. 2011, Nature, 477, 188

Mennesson, B., et al. 2002, ApJ, 579, 446

Menzies, J. W., et al. 2008, MNRAS, 385, 1045

Menzies, J. W., et al. 2010, MNRAS, 406, 86

Menzies, J. W., et al. 2011, MNRAS, 414, 3492

Pietrzyński, G., et al. 2013, Nature, 495, 76

Rich, R. M. 2013, Planets, Stars and Stellar Systems Vol. 5, Dordrecht: Springer, p. 273

Reid, M. J., et al. 2009, ApJ, 705, 1548

Schönrich, R., et al. 2011, MNRAS, 415, 3807

Sloan, G., et al. 2012, ApJ, 752, 140

Soszyński, et al. 2008, AcA, 58, 293

Stepanishchev, A. S., \& Bobylev, V. V. 2013, Ast. L., 39, 185

van Leeuwen, F., et al. 2007, MNRAS, 379, 723

Vásquez, S., et al. 2013, A\&A, in press, arXiv1304.6427

Whitelock, P. A., Feast, M. W., \& Catchpole, R. M. 1991, MNRAS, 248, 276

Whitelock, P. A., \& Catchpole, R. 1992, The Center, Bulge, and Disk of the Milky Way, Dordrecht: Kluwer, p. 103

Whitelock, P. A., et al. 1997, MNRAS, 288, 512

Whitelock, P. A., et al. 2006, MNRAS, 369, 751

Whitelock, P. A., Feast, M. W., \& van Leeuwen, F. 2008, MNRAS, 386, 313

Whitelock, P. A., et al. 2009, MNRAS, 394, 795

Whitelock, P. A., et al. 2013, MNRAS, 428, 2216

Windmark, F., Lindegren, L., \& Hobbs, D. 2011, A\&A, 530, 76

\section{Discussion}

ŽELJKO IVEzIĆ: Could you please summarize physical reasons for the age-period relation for Miras, and comment on whether this relation depends on metallicity?

PATRicia Whitelock: It is difficult to answer this fully because all theoretical work depends on assumptions about mass loss at the tip of the AGB where the Miras lie. The observations we discussed indicate that such a relation exists though we do not have a very clear idea of its scatter, i.e. the range of periods at a given age (and metallicity). As regards metallicity dependence, we know that in globular clusters the Mira period is larger for more metal-rich clusters (e.g., Feast \& Whitelock 2000). This might for instance be due to atmospheric structure affecting the stellar radius, but that is not clear. Further work on the age-period relation is desirable, especially for C-Miras. However, $\mathrm{C}$ and 
O-Miras have rather similar absolute magnitudes at the same period and probably similar ages.

LiCAI Deng: Are those outliers in the two PLs the same? Is there any physical reason for them to be outliers?

PATRICIA Whitelock: They are either undergoing line-of-site obscuration (see section 6.2) or the bolometric correction does not apply to these short period dusty stars, which seem unique to the dwarf spheroidals. 